TITLE:

\title{
Synergic effects of 9,10- phenanthrenequinone and cadmium on pro-inflammatory responses in airway epithelial cells
}

\section{AUTHOR(S):}

Honda, Akiko; Chowdhury, Pratiti Home; Ito, Sho; Okano, Hitoshi; Onishi, Toshinori; Kawaryu, Yusuke; Ueda, Kayo; Takano, Hirohisa

\section{CITATION:}

Honda, Akiko ...[et al]. Synergic effects of 9,10-phenanthrenequinone and cadmium on pro-inflammatory responses in airway epithelial cells. Environmental Toxicology and Pharmacology 2017, 52: 276-279

\section{ISSUE DATE:}

2017-06

URL:

http://hdl.handle.net/2433/243827

\section{RIGHT:}

(c) 2017. This manuscript version is made available under the CC-BY-NC-ND 4 o license

http://creativecommons.org/licenses/by-nc-nd/4.0/; The full-text file will be made open to the public on 1 June 2018 in accordance with publisher's 'Terms and Conditions for Self-Archiving'.; This is not the published version. Please cite only the published version.; この論文は出版社版でありません。引用の際には出版社版をご確認で利用ください。 
Short Communication

Synergic effects of 9,10-phenanthrenequinone and cadmium on pro-inflammatory responses in airway epithelial cells

Akiko Honda ${ }^{\dagger} *$, Pratiti Home Chowdhury ${ }^{\dagger}$, Sho Ito ${ }^{\dagger}$, Hitoshi Okano ${ }^{\dagger}$, Toshinori Onishi ${ }^{\dagger, \ddagger}$, Yusuke Kawaryu ${ }^{\dagger}$, Kayo $\mathrm{Ueda}^{\dagger}$ and Hirohisa Takano ${ }^{\dagger}$

${ }^{\dagger}$ Environmental Health Division, Department of Environmental Engineering, Graduate School of Engineering, Kyoto University. Kyoto, Japan

${ }^{\ddagger}$ Department of Otolaryngology-Head and Neck Surgery, Kyoto Prefectural University of Medicine, Kyoto, Japan

Running title: PQ and Cd synergistically affect pro-inflammatory responses

*Corresponding author: Dr. Akiko Honda, Environmental Health Division, Department of Environmental Engineering, Graduate School of Engineering, Kyoto University, C Cluster, Kyoto-Daigaku-Katsura, Nishikyo-ku, Kyoto 615-8540, Japan. Tel.: +81-75-383-3345; Fax: +81-75-383-3344.

E-mail: akko@health.env.kyoto-u.ac.jp 


\section{Abstract}

We investigated the synergic effects of components of particulate matter with aerodynamic diameters $\leq 2.5 \mu \mathrm{m}$ (PM2.5) on airway inflammation. Co-exposure to cadmium (Cd) and 9,10-phenanthrenequinone (9,10-PQ) additively/synergistically increased pro-inflammatory responses in airway epithelial cells, whereas co-exposure to $\mathrm{Cd}$ and phenanthrene resulted in no acceleration. These results suggest that the combination of metal and a quinone derivative can contribute to the exacerbation of respiratory diseases by PM2.5.

Key words: cadmium, 9,10-phenanthrenequinone, synergic effects, airway inflammation, PM2.5 


\section{Introduction}

Particulate matter with aerodynamic diameters $\leq 2.5 \mu \mathrm{m}$ (PM2.5) is composed of elemental carbons, organic carbons and metals. It has been demonstrated that singleexposures to each component contribute to respiratory diseases (Inoue et al. 2005; Hiyoshi et al. 2005; Honda et al. 2016). However, considering the constituents of PM2.5, it is important to identify the combined effects of components of PM2.5. Interestingly, it was reported that the interleukin (IL)-6 and IL-8 release from airway epithelial cells caused by organic extracts from PM2.5 were reduced by a metal chelator (RodríguezCotto et al. 2015). That finding indicates that a combination of organic components and metals in PM2.5 may lead to stronger pro-inflammatory responses. However, it is not clear which of the organic and metal components of PM2.5 play critical roles in the combined effects on the exacerbation of respiratory diseases.

Among the PAHs, 9,10-phenanthrenequinone (9,10-PQ) is directly emitted from vehicles and is contained in PM2.5 that includes diesel exhaust particles (Choo et al. 2004). Koike et al. (2014) reported that exposure to 9,10-PQ but not phenanthrene (Phe) induces cytotoxic effects on airway epithelial cells. Hiyoshi et al. (2005) suggested that 9,10-PQ exacerbates the pathogenesis of asthma, with effects on airway inflammation in the presence of ovalbumin (OVA) as an antigen. The deterioration of respiratory health induced by 9,10-PQ in PM2.5 is thus a public health concern.

Among the wide variety of metals, previous research detected cadmium (Cd) in ambient air and has examined the health effects of Cd (Suvarapu et al. 2017). It was also reported that the lead $(\mathrm{Pb})$ and Cd levels in PM2.5 and in study participants' blood were higher in an electronic waste-exposed area, and that the prevalence of respiratory symptoms such as coughing and phlegm were higher (Zeng et al. 2016). Another research group demonstrated that Cd levels in the blood are significantly associated with the pathogenesis of asthma (Park et al. 2016). It is thus possible that Cd in PM2.5 affects airway epithelial cells and can thus exacerbate respiratory diseases.

In the present study, we therefore focused on the combined effects of metal and PAHs (especially Cd and 9,10-PQ) as the components of PM2.5 on airway epithelial cells. We also compared the respiratory effects of 9,10-PQ and those of the parent PAH, i.e., Phe, in the presence and absence of Cd.

\section{Materials and methods}

\subsection{Chemicals}

The quinone derivative i.e. 9,10-PQ and its parent PAH i.e. Phe, were purchased from Sigma (St. Louis, MO, USA) and Tokyo Chemical Industry (Tokyo), respectively. 
Cadmium sulfate hydrate was purchased from Sigma.

\subsection{Experimental protocol}

After the BEAS-2B cell line as airway epithelial cells grew to semi-confluence in LHC-9 medium in collagen I-coated plates, they were exposed to Cd $(0,1,10 \mu \mathrm{M})$, and 9, 10-PQ $(1 \mu \mathrm{M})$ or Phe $(1 \mu \mathrm{M})$ for $3 \mathrm{hr}$ or $24 \mathrm{hr}$. We then evaluated the releases of IL-6 and IL-8 in the culture supernatants, the generation of reactive oxygen species (ROS), and the metallothionein 2A (MT-2A) mRNA expression by performing enzyme-linked immunosorbent assay (ELISA), a CM- $\mathrm{H}_{2}$ DCFDA fluorescent probe, and a real-time polymerase chain reaction (RT-PCR), respectively. Our previous study showed that extracts of PM2.5 collected from two areas in Japan induced IL-6 release from airway epithelial cells under nontoxic conditions (Honda et al. in press), and we therefore used lower doses of Cd, 9,10-PQ and Phe without cytotoxicity in the present experiment (Suppl. Fig. S1A,B).

\subsection{Quantitation of inflammatory proteins in the culture supernatants}

After exposure for $24 \mathrm{hr}$, the levels of IL-6 and IL-8 in the culture medium were measured by ELISA (Thermo Scientific, Waltham, MA) according to the manufacturer's instructions. The detection limits of IL-6 and IL-8 were $<1 \mathrm{pg} / \mathrm{mL}$ and $<2 \mathrm{pg} / \mathrm{mL}$, respectively.

\subsection{ROS generation}

We used a fluorescent probe, 5-(and-6)-chloromethyl-2',7'dichlorodihydrofluorescein diacetate, acetyl ester (CM- $\mathrm{H}_{2} \mathrm{DCFDA}$ ), to measure the intracellular ROS generation. The fluorescence intensity during 0-3 hr (excitation 485 $\mathrm{nm}$, emission $530 \mathrm{~nm}$ ) was measured.

\subsection{Extraction of RNA and quantitative RT-PCR analysis}

After exposure for $3 \mathrm{hr}$, total RNA was extracted with an RNeasy Mini Kit (Qiagen, Hilden, Germany) and was reverse-transcribed to cDNA using a High Capacity RNA-to-cDNA kit (Life Technologies) according to the manufacturer's instructions. The quantitation of mRNA expression was carried out using the ABI Prism 7000 Sequence Detection System (Life Technologies). The relative intensity was normalized to $\beta$-ACTIN as an endogenous control gene. TaqMan probes and pair primers for MT-2A and $\beta$ - 
ACTIN were designed by Life Technologies, which does not disclose these sequences.

\subsection{Statistical analysis}

Data are presented as mean \pm standard error of the mean (SEM) for each experimental group $(n=4)$. Differences among groups were analyzed using the Tukey multiple comparison test (Excel Statistics 2010, Social Survey Research Information, Tokyo). A $p$-value $<0.05$ was accepted as significant.

\section{Results}

3.1 The combined effects of Cd and 9,10-PQ or Phe on the cytokine production

Both, Cd at a dose of $10 \mu \mathrm{M}$ and 9,10-PQ alone increased the protein release of IL6 compared to the controls (Fig. 1.A). Cd in the presence of 9,10-PQ also induced the release of IL-6 compared to the controls. Cd at the doses of 1 or $10 \mu \mathrm{M}$ in the presence of 9,10-PQ elevated the protein release of IL-6 compared to Cd alone at the same doses. IL6 release was most highly induced by $\mathrm{Cd}$ at the dose of $10 \mu \mathrm{M}$ in the presence of 9,10PQ.

No change in IL-6 release was observed after Phe-alone exposure (Fig. 1.B). Cd at the dose of $10 \mu \mathrm{M}$ in the presence of Phe also increased the IL-6 release compared to the controls. The degree of increase by $\mathrm{Cd}$ with Phe was almost the same as that by $\mathrm{Cd}$ alone.

$\mathrm{Cd}$ at $10 \mu \mathrm{M}$ increased the protein release of IL-8 compared to the controls (Fig. 1C). No change in IL-8 release was observed after 9,10-PQ-alone exposure. Cd in the presence of 9,10-PQ induced the release of IL-8 compared to the controls. Cd at the dose of $10 \mu \mathrm{M}$ in the presence of 9,10-PQ elevated the protein release of IL-8 compared to Cd alone at the same dose.

No change in IL-8 release was observed after Phe-alone exposure (Fig. 1.D.). Cd at $10 \mu \mathrm{M}$ in the presence of Phe increased the IL-8 release compared to the controls. The degree of increase caused by $\mathrm{Cd}$ with Phe was almost the same as that by $\mathrm{Cd}$ alone.

\subsection{The combined effects of Cd and 9,10-PQ or Phe on oxidative stress}

We observed that both 9,10-PQ alone and Cd plus 9,10-PQ generated ROS after 20 min of exposure (Fig. 2.A). At $3 \mathrm{hr}$ after exposure, the level of ROS induced by Cd alone was similar to that of the controls, whereas 9,10-PQ alone greatly increased ROS compared to the controls (Fig. 2.B). Cd in the presence of 9,10-PQ also induced the generation of ROS compared to the controls, but the increased levels after co-exposure to Cd plus 9,10-PQ were similar to those after 9,10-PQ alone. No change in ROS generation was observed after Phe-alone exposure or Cd with Phe exposure (Suppl. Fig. S2). 
Cd alone elevated the MT-2A expression compared to the controls, whereas 9,10PQ alone did not increase the MT-2A expression compared to the controls (Fig. 2.C). Cd in the presence of 9,10-PQ also induced MT-2A compared to the controls, whereas the increased levels after co-exposure to Cd plus 9,10-PQ were similar to those obtained with Cd alone.

\section{Discussion}

To our knowledge, this is the first report that co-exposure to Cd plus 9,10-PQ additively or synergistically enhanced pro-inflammatory responses. An earlier study suggested that Cd alone induces IL-6 and IL-8 production from airway epithelial cells, which is in accord with our present results (Rennolds et al. 2012). It was also reported that 9,10-PQ exacerbated the pathogenesis of asthma in the presence of ovalbumin (OVA) (Hiyoshi et al. 2005). Our present findings indicated that Cd has stronger effects on IL-6 and IL-8 releases than 9,10-PQ under non-toxic conditions. In light of the results of previous and the present studies, it appears that 9,10-PQ can act mainly as a positive adjuvant of pro-inflammatory responses under co-exposure of Cd and 9,10-PQ in airway epithelial cells.

In contrast to 9,10-PQ, Phe did not affect the Cd-induced pro-inflammatory responses. The difference between 9,10-PQ and Phe is their ability of ROS generation. We therefore measured the intracellular ROS, but we observed no change in the generation of ROS after a 3-hr exposure to Cd plus 9,10-PQ compared to 9,10-PQ alone. These results indicate that the generation of ROS induced by 9,10-PQ does not enhance Cd-induced pro-inflammatory responses.

Cysteine rich-metallothionein (MT) plays a key role in the detoxification of Cd by forming a Cd-MT complex, and MT also has antioxidant capacity (Satoh 2007). A decrease of MT has the potential to exacerbate Cd toxicity or Cd-induced airway inflammation. However, we were not able to confirm the inhibition of MT expression by 9,10-PQ. In the present study, MT was similarly induced by $\mathrm{Cd}$ in the presence and absence of 9,10-PQ, which may indicate that 9,10-PQ does not affect the MT expression.

It has been reported that the activation of nuclear factor- $\kappa \mathrm{B}$, extracellular signalregulated kinase 1/2, activator protein-1, or EGF-R signal transduction pathways by Cd leads to pro-inflammatory responses (Kundu et al. 2011; Cormet-Boyaka et al. 2012; Olszowski et al. 2012). Therefore, Cd with 9,10-PQ may stimulate these transcription factors more heavily than $\mathrm{Cd}$ alone and thus prompt pro-inflammatory responses. In addition, it was suggested that metals and PAHs have the potential to form metal-PAH complexes (Gauthier et al. 2014). A Cd-9,10-PQ complex as a new chemical substance 
may react with the cell surface or intracellular molecules and then prompt IL-6 and IL-8 releases. Further investigation is needed to identify the mechanisms by which 9,10-PQ increases Cd-induced pro-inflammatory responses in airway epithelial cells.

In conclusion, Cd and 9,10-PQ as components of PM2.5 additively/synergistically increase pro-inflammatory responses, which can contribute to the exacerbation of respiratory diseases.

\section{Conflict of interest}

The authors declare that there are no conflicts of interest.

\section{Acknowledgments}

This study was supported by Grants-in-Aid for Scientific Research in Japan (Grant no. 16K19272). We are grateful for Tomohiro Hayashi's contribution at the preliminary stages of this work. We thank Megumi Nagao for the data analysis support, and we thank Kiyoe Itoi and Yufuko Kobayashi for their technical assistance. 


\section{References}

Cho AK, Stefano ED, You Y, Rodriguez CE, Schmitz DA, Kumagai Y, Miguel AH, Eiguren-Fernandez A, Kobayashi T, Avol E, Froines JR. 2004. Determination of four quinones in diesel exhaust particles, SRM 1649a, and atmospheric PM2.5. Special Issue of Aerosol Science Technology on Findings from the Fine Particulate Matter Supersites Program. Aerosol Sci Technol. 38:68-81.

Cormet-Boyaka E, Jolivette K, Bonnegarde-Bernard A, Rennolds J, Hassan F, Mehta P, Tridandapani S, Webster-Marketon J, Boyaka PN. 2012. An NF-кB-independent and Erk1/2-dependent mechanism controls CXCL8/IL-8 responses of airway epithelial cells to cadmium. Toxicol Sci. 125:418-29.

Gauthier PT, Norwood WP, Prepas EE, Pyle GG. 2014. Metal-PAH mixtures in the aquatic environment: A review of co-toxic mechanisms leading to more-than-additive outcomes. Aquat Toxicol. 154:253-269.

Hiyoshi K, Takano H, Inoue KI, Ichinose T, Yanagisawa R, Tomura S, Kumagai Y. 2005. Effects of phenanthraquinone on allergic airway inflammation in mice. Clin Exp Allergy. 35:1243-8.

Honda A, Tsuji K, Matsuda Y, Hayashi T, Fukushima W, Sawahara T, Kudo H, Murayama R, Takano H. 2015. Effects of air pollution-related heavy metals on the viability and inflammatory responses of human airway epithelial cells. Int J Toxicol. 34:195-203.

Honda A. Fukushima W, Oishi M, Tsuji K, Sawahara T, Hayashi T, Kudo H, Kashima Y, Takahashi K, Sasaki H, Ueda K, Takano H. Effects of components of PM2.5 collected in Japan on the respiratory and immune systems. Int $J$ Toxicol. in press. doi: 10.1177/1091581816682224.

Inoue K, Takano H, Yanagisawa R, Sakurai M, Ichinose T, Sadakane K, Yoshikawa T. 2005. Effects of nano particles on antigen-related airway inflammation in mice. Respir Res. 6:106.

Koike E, Yanagisawa R, Takano H. 2014. Toxicological effects of polycyclic aromatic hydrocarbons and their derivatives on respiratory cells. Atmos Environ. 97:529536.

Kundu S, Sengupta S, Bhattacharyya A. 2011. EGFR upregulates inflammatory and proliferative responses in human lung adenocarcinoma cell line (A549), induced by lower dose of cadmium chloride. Inhal Toxicol. 23:339-48.

Olszowski T, Baranowska-Bosiacka I, Gutowska I, Chlubek D. 2012. Pro- 
inflammatory properties of cadmium. Acta Biochim Pol. 59:475-82.

Park S, Lee EH, Kho Y. 2016. The association of asthma, total IgE, and blood lead and cadmium levels. J Allergy Clin Immunol. 138:1701-1703.e6.

Rennolds J, Malireddy S, Hassan F, Tridandapani S, Parinandi N, Boyaka PN, Cormet-Boyaka E. 2012. Curcumin regulates airway epithelial cell cytokine responses to the pollutant cadmium. Biochem Biophys Res Commun. 417:256-61.

Rodríguez-Cotto RI, Ortiz-Martínez MG, Jiménez-Vélez BD. 2015. Organic extracts from African dust storms stimulate oxidative stress and induce inflammatory responses in human lung cells through Nrf2 but not NF-кB. Environ Toxicol Pharmacol. 39:845-856.

Satoh M. 2007. Analysis of toxicity using metallothionein knockout mice. Yakugaku Zasshi. 127:709-17.

Suvarapu LN, Baek SO. 2017. Determination of heavy metals in the ambient atmosphere: A review. Toxicol Ind Health 33:79-96. doi: 10.1177/0748233716654827.

Zeng X, Xu X, Zheng X, Reponen T, Chen A, Huo X. 2016. Heavy metals in PM2.5 and in blood, and children's respiratory symptoms and asthma from an e-waste recycling area. Environ Pollut. 210:346-53. 


\section{Figure legends}

Fig. 1. Combined effects of Cd plus 9,10-PQ (A, C) or Phe (B, D) on the IL-6/IL-8 production from airway epithelial cells. ${ }^{* *} p<0.01$ vs. $0 \mu \mathrm{M} \mathrm{Cd}$ in the absence of PAHs, ${ }^{\S} p<0.05,{ }^{\S} p<0.01$ vs. each other.

Fig. 2. Combined effects of Cd plus 9,10-PQ on oxidative stress in airway epithelial cells. The intracellular levels of ROS during the 3-hr exposure (A) and at $3 \mathrm{hr}$ (B) and the expression of MT-2A mRNA at $3 \mathrm{hr}(\mathbf{C})$ after exposure were measured by a CM$\mathrm{H}_{2}$ DCFDA fluorescent probe and RT-PCR, respectively. ${ }^{*} p<0.05,{ }^{* *} p<0.01$ vs. $0 \mu \mathrm{M} \mathrm{Cd}$ in the absence of 9,10-PQ, ${ }^{\S \S} p<0.01$ vs. each other.

Fig.1
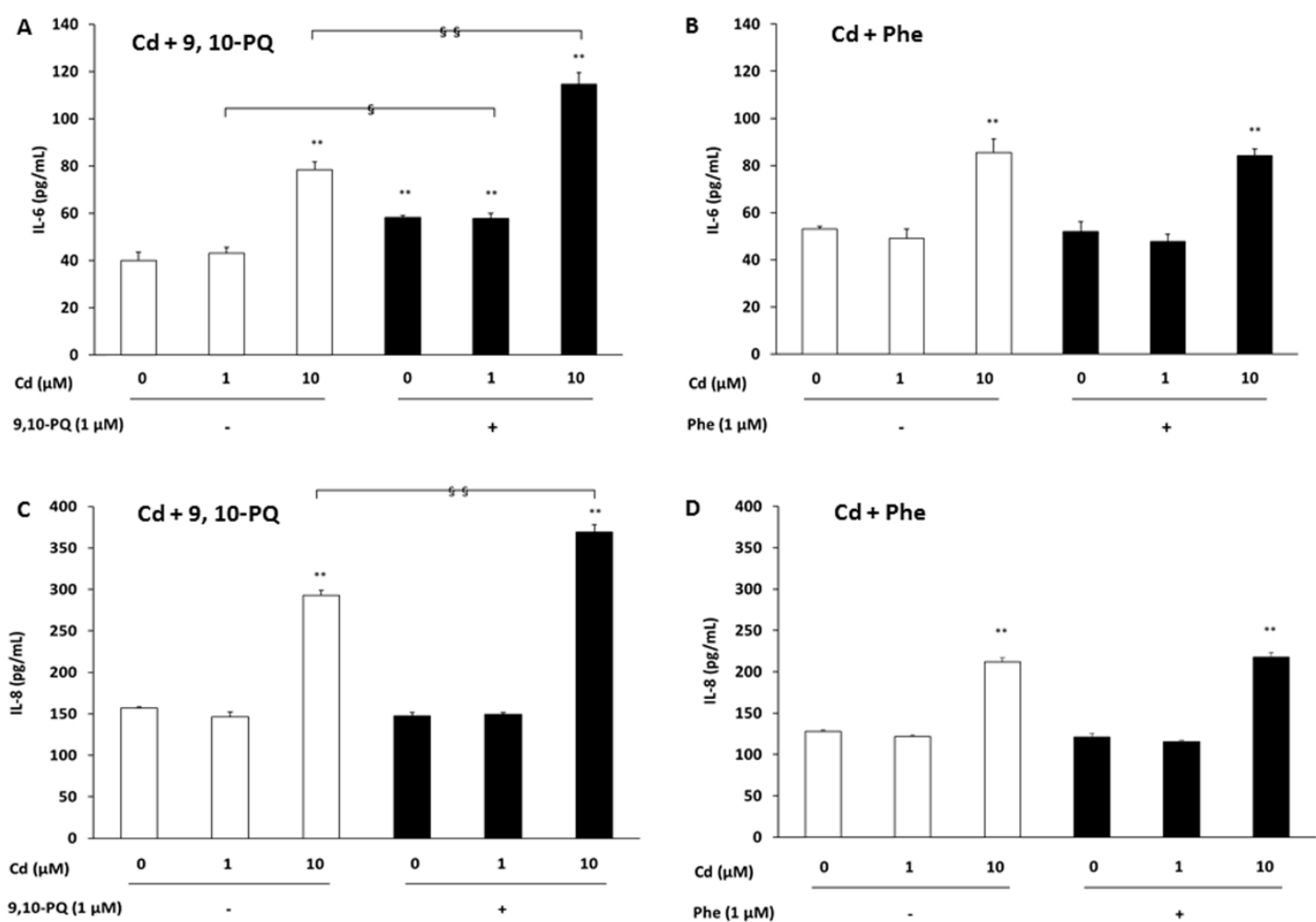
Fig.2

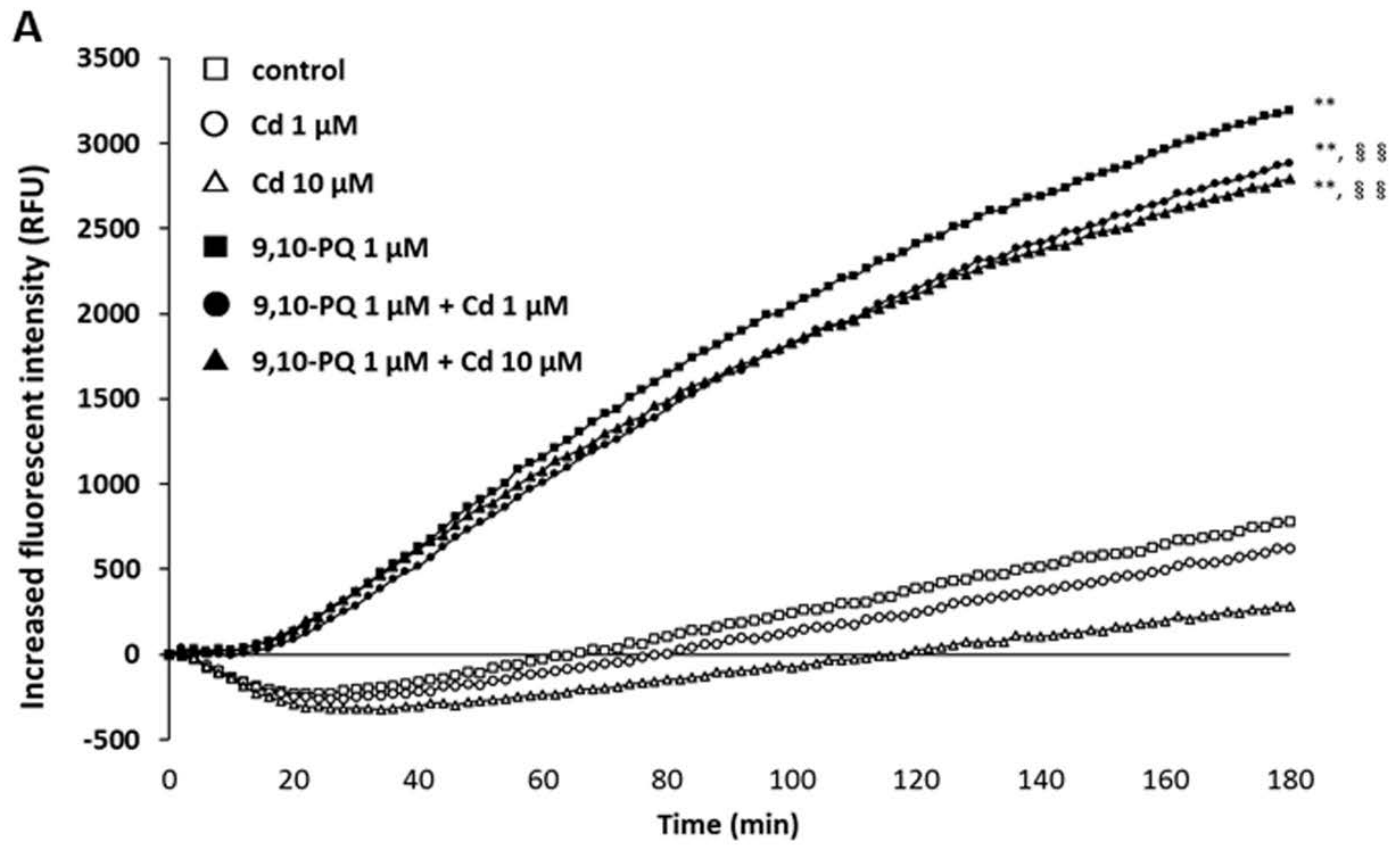

B

$180 \mathrm{~min}$

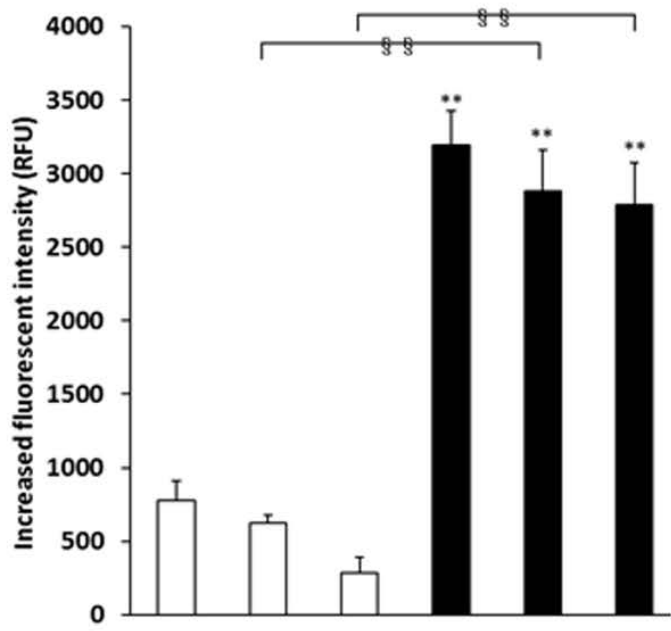

$\mathrm{Cd}(\mu \mathrm{M})$

9,10-PQ (1 $\mu \mathrm{M})$
C

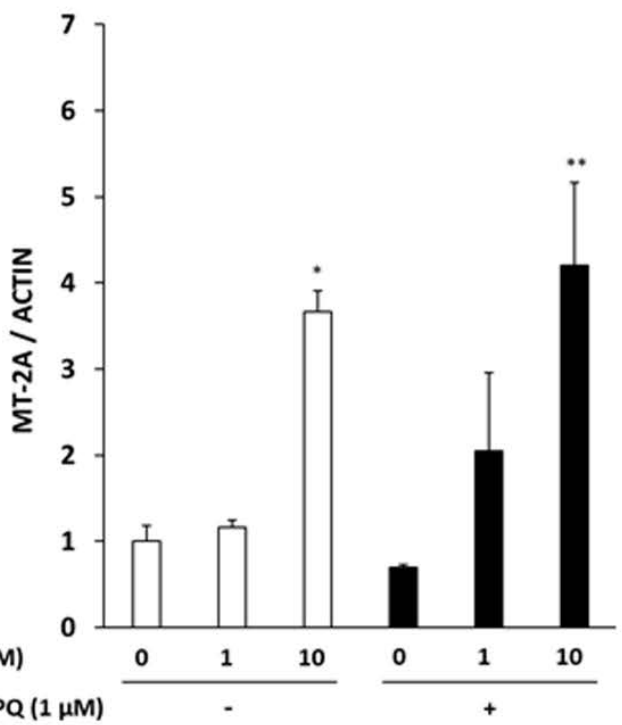


A

$C d+9,10-P Q$

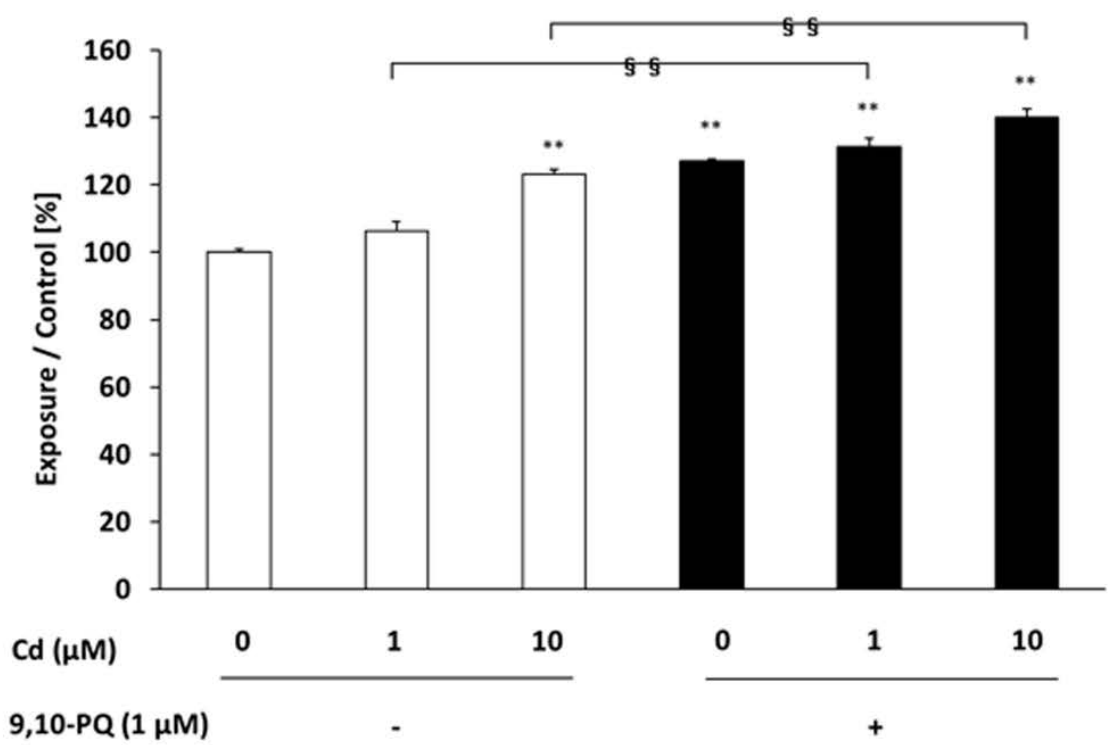

B

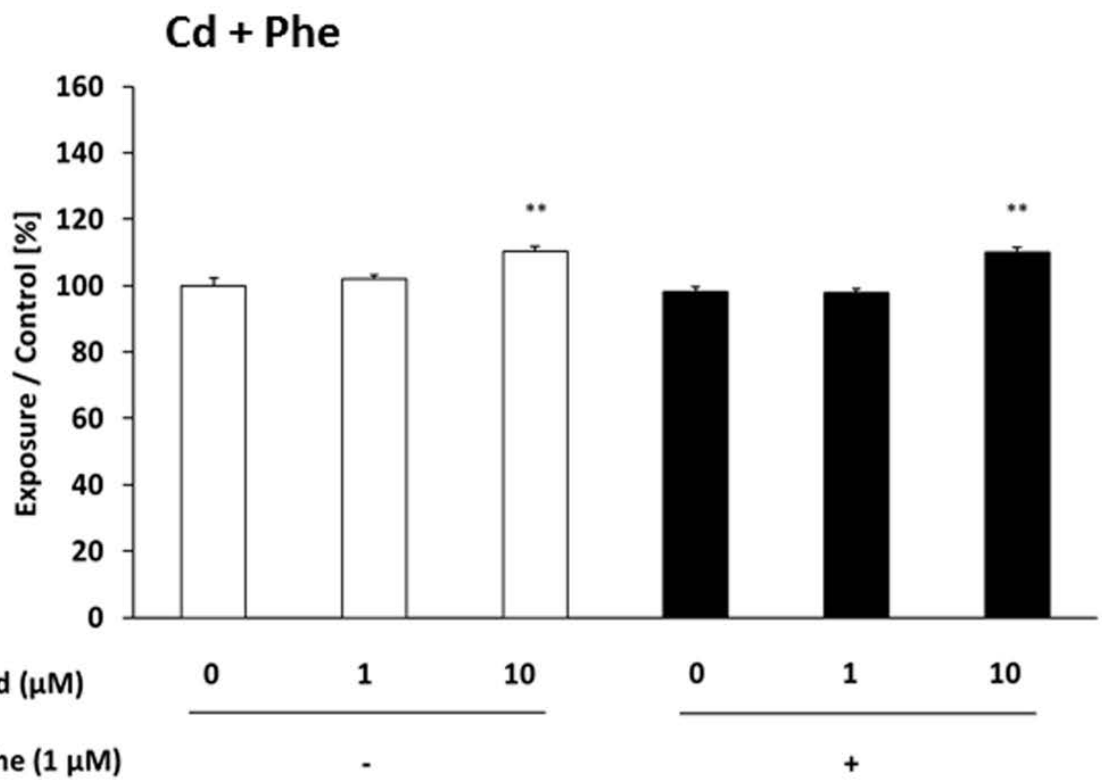

Suppl. Fig. S1. Combined effects of Cd plus 9,10-PQ (A) or Phe (B) on the viability of airway epithelial cells. Cell viability was assessed by WST-1 assays. Cells were treated with the indicated concentrations for $24 \mathrm{hr}$. The data are presented as the percentage of the viability of the control. ${ }^{* *} p<0.01$ vs. $0 \mu \mathrm{MCd}$ in the absence of PAHs, ${ }^{\widehat{ }} \widehat{\S} p<0.01 \mathrm{vs}$. each other. 


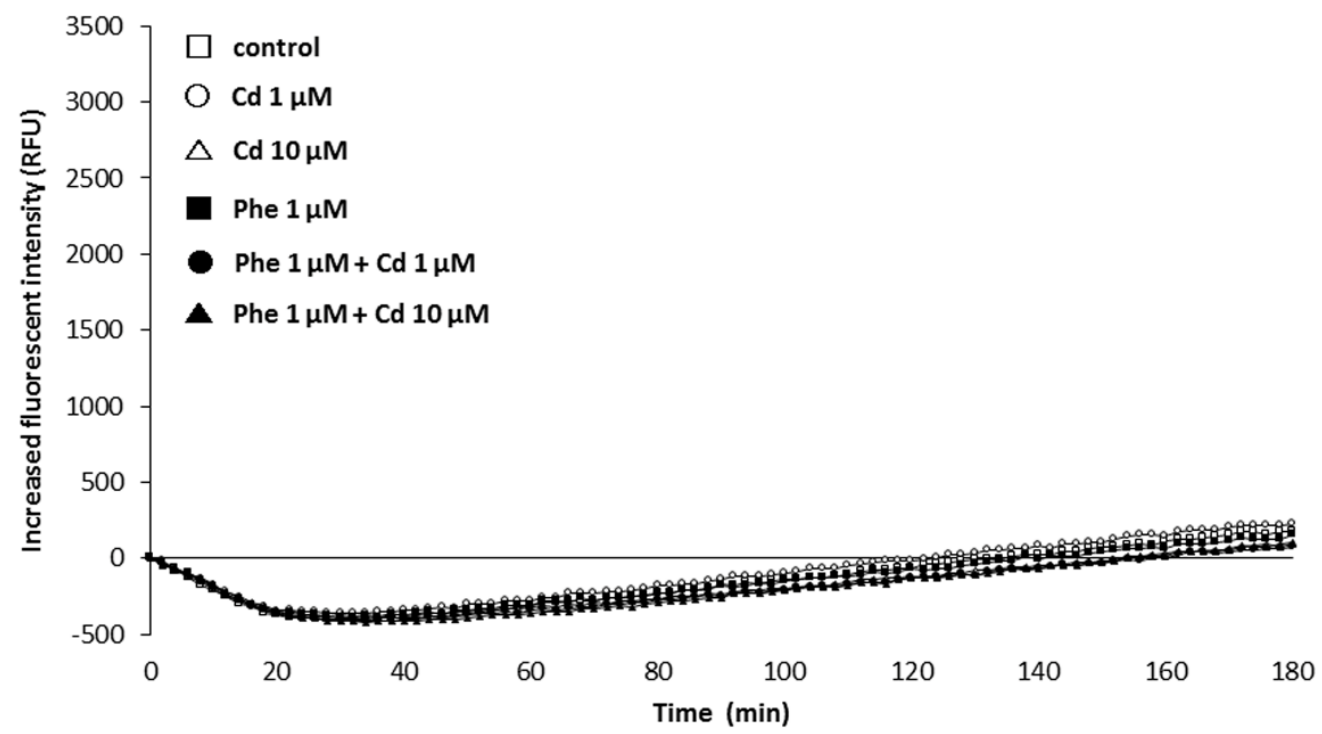

Suppl. Fig. S2. Combined effects of Cd plus Phe on the intracellular levels of ROS. The ROS level was assessed with a CM- $\mathrm{H}_{2} \mathrm{DCFDA}$ fluorescent probe. Cells were treated with the indicated concentrations for $3 \mathrm{hr}$. 